\title{
COMPARATIVE ANALYSIS OF DSDV, DSR, AODV WITH GPSR IN VANET SCENARIO
}

\author{
KANISHKA RAHEJA ${ }^{1} \&$ AMIT KUMAR GOEL ${ }^{2}$ \\ ${ }^{1}$ Scholar, Computer Science and Engineering, SGT University, Gurugram, Haryana, India \\ ${ }^{2}$ Professor, School of Computing, Science and Engineering, Galgotias University, Greater Noida, Uttar Pradesh, India

\begin{abstract}
VANET is Mobile Adhoc Network in which Vehicles are dynamic in Nature and topology changes frequently. Hence, routing is a big challenge in VANET as Vehicles themselves act as nodes in Network. VANETs have many networking research challenges, and therefore, the design of a protocol with much efficiency is essential. Various protocols have been designed to minimize this challenge. Therefore the performances of Vehicle to Vehicle and Vehicle to roadside communication depend on various protocols. In this paper we present comparative analysis of different Topology based Routing protocols such as AODV, DSDV and DSR with GPSR. The performance of these protocols is examined on the basis of NS-2.35 simulations by using different parameters such as Throughput, PDR, Routing Overhead, End to End Delay and energy for different number of vehicles.

KEYWORDS: VANET,NS-2,AODV,DSR,DSDV,ITS,RSU, OBU \& PDR
\end{abstract}

Received: May 13, 2020; Accepted: Jun 03, 2020; Published: Aug 01, 2020; Paper Id.: IJMPERDJUN2020553

\section{INTRODUCTION}

Need of an Intelligent transport system emerged as according to report published by World Health Organization, the deaths and injuries at the road traffic caused by accidents are due to driver carelessness, population, violation of traffic rules, congestion and many more situations.[1] Therefore there must be intelligent transport facility to not undergo the setypes of accidents. The aim of ITS is to enhance traffic flow and to provide traffic safety and For reducing traffic congestion and reduce road accidents VANET is an advanced network technology solution that offers Intelligent Transportation services to end users.[2] VANET is type of MANET which depends up on registration mechanism, road side units(RSU) and on Board Units(OBU). OBU are installed on every vehicle to communicate with another vehicle while Road side units are used to communicate with infrastructure and contain network devices for dedicated short range communications.[3] VANET is to be classified into two categories-Vehicle to Vehicle Communications and Vehicle to Infrastructure communications. The main responsibility of VANET is to produce effective communications because the nodes need to acquire information by communicating with neighbors and then to take decisions accordingly by information collected by using cameras, sensors, GPS(Global Positioning Systems) receivers and omnidirectional antennas.[4]

As the nodes or vehicles in case of VANET are mobile in nature, move in high speed and structure changes frequently, thus routing of data packets becomes difficult in case of VANET. VANETs are highly dynamic as compared to other Adhoc based Networks because every vehicle is equipped with GPS and has its own geogrophic location and storage space and battery power are not limited. [5] 


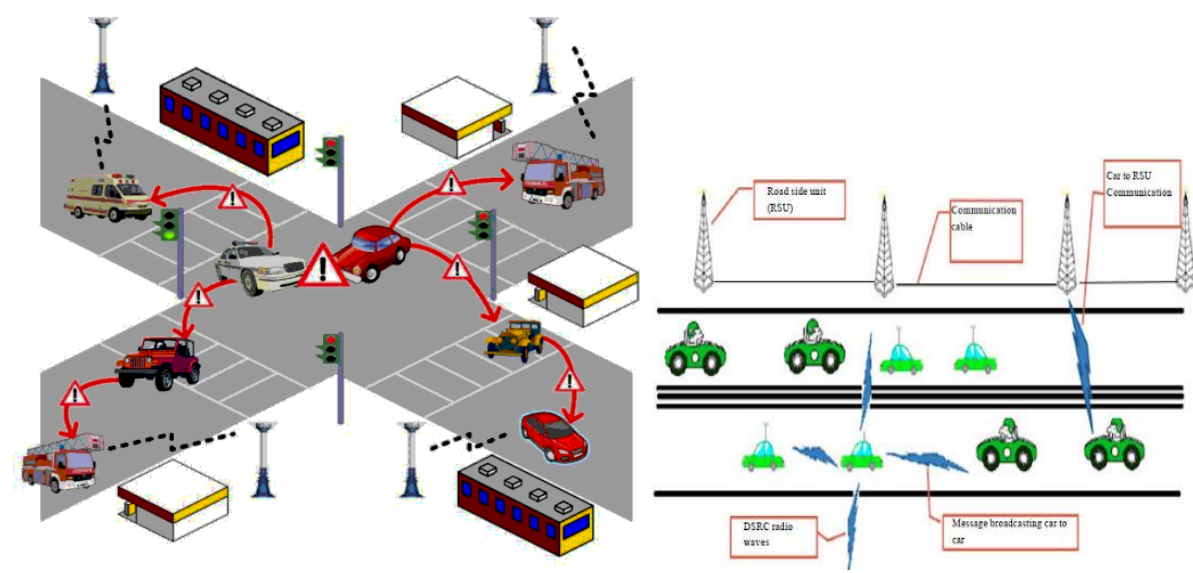

Figure 1: a. Vehicle to Vehicle Communic1ation -Vehicle to Road Side Communication b. VANET Scenario.

Routing Protocols of VANET's is divided into 5 categories:

- Topology Based

- Cluster Based

- Position Based

- Broadcast Based

- Infrastructure Based

- Geocast based

VANET deals with two wireless access standards: (i) IEEE 802.11 manages the physical and MAC Layer (ii) IEEE 1609 manages the higher Level Protocol. In addition, to efficiently detect route from source to destination, AODV and DSR are considered as routing protocols. [6]In the literature, comparative analysis of AODV, DSR and DSDV with GPSR protocol will be observed. The main contribution of the paper includes the following:

Survey on the aforesaid protocols and brief introduction about the protocols based on the survey. In the second step analyzing the impact of wireless channel on throughput, PDR, Routing Overhead, E to E delay of VANET. In the last step, a set of simulations has been designed and performed to evaluate the overall performance parameters such as throughput, energy, Routing overhead, End to End Delay and Packet Delivery Ratio using different number of nodes on different Routing protocols mentioned above.

Rest of the paper is organized as follows: Section 2 will give introduction of Simulation Setup, description of the protocols on which simulation has to be done and wireless channel model. Section 3 describes all perimeters on which the analysis has to be done. Results and discussions will be described in section 4of the paper\& finally section 5 describes conclusion and future work.

\section{MODEL}


In our work, we have created various realistic traffic scenarios and Road side units (RSU) in which vehicle to vehicle and vehicle to Road side units are shown. Different results are analysed by increasing the number of vehicles on the roads. Work of simulation is being done with NS-2.35 simulator and IEEE802.11 MAC Protocol is being used in our work. It further includes that packet size of 1500 Bytes is used. Table 1 shows all the perimeters used in the work

Table 1: Simulation Parameters

\begin{tabular}{|l|l|}
\hline Simulation Parameters & \multicolumn{1}{|c|}{ Value } \\
\hline Network Simulator & NS-2.35 \\
\hline Routing Protocol & AODV,DSDV,DSR,GPSR \\
\hline Simulation Area & $500 \mathrm{~m}$ x500m, change according to requirement \\
\hline Number of Nodes & $10,20,30,40,50$ change according to requirement \\
\hline MAC Protocol & IEEE802.11 \\
\hline Simulation Time & 50 sec. change according to requirement \\
\hline Connection Type & TCP \\
\hline Packet Size & 1500 Bytes \\
\hline Data Rate & $1 \mathrm{Mbps}$ \\
\hline Pause Time & 0.5 sec. change according to requirement \\
\hline Nodes Speed & $10 \mathrm{~m} /$ sec. change according to requirement \\
\hline Antenna & Antenna/OmniAntenna \\
\hline Propagation Model & Propagation/TwoRayGround \\
\hline Channel & Channel/WirelessChannel \\
\hline
\end{tabular}

\subsection{Routing Protocols}

Major issue in VANET as described before is of Routing. For providing efficient communication in V2V and V2I Environment in AdHoc Networks, many Routing protocols have been reported so far [7]. The principle issues in Vehicular Adhoc Networks which need routing protocols are, Quality of Service, Mobility, Quick Information Exchange and so on. In our Scenario we are adopting already existing three Topology based Protocols i.e. AODV, DSR, DSDV are being compared with Position Based Routing Protocols GPSR which is also an existing Protocol.

AODV (Ad Hoc on Demand Distance Vector Routing Protocol): It is an On Demand Reactive Protocol for wireless networks which uses conventional way of using tables to store routing data. All possible paths from one node to another will be stored in Routing tables. This protocol uses timer for every mobile node and if the particular route is not utilized for specific period of time, it discards the entry from table. Firstly, Route discovery phase in this protocol follows a Route Request and Route Reply Cycle. Route Error (RERR) message is broadcasted which contains all the destinations on which the link is not available. Route maintaining stage includes sending a route request message (RREQ) from communicating node to its neighbors. [8]

DSR (Dynamic Source Routing):It is also an On Demand Reactive Protocol because it discovers the route on Demand as and when required and needed from Source to Destination. In this sense, it is same as AODV. At every intermediate node it looks forward to Source Routing rather than looking forward to Routing Table. The feature which differs from alternate On Demand routing is that it does not find need to transmit the message periodically to its neighbors. DSR has two phases:

- Route Searching: To perform Route searching REQP message will be sent.

- Route Maintenance

There are some rules which each and every node has to follow: 
- If any destination node has received REQP only then it can receive packet.

- If packet has been received by a node which is not destination node it adds its own information and then it will transmit the packet.

- If at any destination node identical packet has arrived, it drops the packet.

When REQP reaches destination after traversing all nodes a REPR Packet will be sent through the same path discovered by REQP packets. If any error is arrived at any node then corresponding node sends a message to the source node by sending route error packet. [9][10]

DSDV (Destination Sequence Distance Vector Routing)-It is a Proactive Protocol which comes under proactive category of Routing Protocols. It maintains table of information in the presence of any other node in the network. If there is any change in the routing Network, it will be communicated to all other nodes in the network and simultaneously it updates the table periodically. Change in route will be broadcasted throughout the network. Routing table which each node associates with it contains: destinations, number of hops to reach destination and the order number corresponding to destination node. A route whose destination number is larger is a new direction and this must be used to create loop avoidance so that always routes that have larger order number are used.[11]

GPSR (Greedy Perimeter Stateless Routing):GPSR is a novel routing protocol for wireless networks, out of the above mentioned protocols. It uses the positions of routers by Global Positioning System and a packet's destination, to make packet forwarding decisions. By using only information about router's immediate neighbors in the network topology, GPSR uses Greedy forwarding decisions. When a packet reaches a region where greedy forwarding is not possible, then the packets are forwarded through Perimeter forwarding and the algorithm recovers by routing around the perimeter of the region. [11][12].

Routing in GPSR is done by two means: Greedy Forwarding and Perimeter Forwarding.

Under GPSR, originator marks the packets with their destination's locations. As a result, a forwarding node can make a greedy choice in choosing the packet's next hop. Specifically, if a node knows its radio neighbors positions then the packet must be forwarded to the immediate neighbor, which is geographically closest to the destination. Forwarding in this system, follows successively closer geographic hops, until the destination is reached.[11][12]

Perimeter Forwarding is done where Greedy Forwarding is not Possible. In Perimeter forwarding packets will be forwarded according to right Hand Rule and this stage is known as recovery mode also.

\subsection{Network interfacing protocols}

Research and Development activities, over the last few decades, of academic and industry leads to the development of IEEE 1609 WAVE and IEEE 802.11 protocol standard which is primarily used to characterize the physical and MAC layers. In our work we have used IEEE 802.11 PHY/MAC protocol [10].

\section{ANALYSIS}

Whenever the research is done it is important to analyze the work which can be presented as: 
1. How many packets are getting delivered from source to destination 2 . How fast the messages can be disseminated to all active vehicles. The most important thing is whether the messages are delivered to all intended nodes.

\subsection{Average Throughput}

Average throughput is specific to a wireless or wired link. In VANET scenario we are using wireless scenario and sharing link. [10]

Throughput $(\mathrm{Mbps})=$ number of bytes transferred in the link $\mathrm{x} 8 /$ total simulation time $(\mu \mathrm{sec})$

\subsection{Packet Delivery Ratio (PDR)}

PDR should have the larger value. It is the percentage of vehicles that are successfully received by all specified vehicles. It is the ratio of the packets which are received by destination to the packets which were sent by source.For better performance of any protocol the packet delivery ratio should be higher [10]

PDR = Packets received successfully/ Total Transmitted packets

\subsection{Average End to End Delay}

It is the time taken by packets to successfully pass through all destination vehicles. Lower the average end to end delay larger is the performance of protocol. It is calculated as follows:

Average EED = Average time taken to deliver packets / Total number of packets sent.

\subsection{Routing Overhead}

Routing overhead is the number of routing packet required for destination. It is defined as follows:

Routing Overhead= total packet size of control packets including RREQ, RREP, RERR and hello packet/ total packet size of data packets delivered to the destination

\subsection{Average Energy}

Average energy consumed by nodes is the mean of energies consumed by each node. Larger the energy consumed by nodes less is the performance of protocol.

\section{RESULTS AND DISCUSSIONS}

In this section we have presented the results by varying the number of vehicles on the road for each of the performance parameter as described in previous section using Network Simulator 2.35 tool for analysis and plotting.

\subsection{Impact of Routing Protocols on Throughput}

Figure 2 shows the impact of throughput on Routing Protocols. During Simulations we have fixed the simulation time to 50 sec and number of nodes is varying from 10 to 50. It is depicted that DSR protocol shows high results of throughput than rest of three protocols. It can also be seen that initially all the four protocols were having similar value of throughput then, DSR and GPSR rised when the number of nodes were increased. Different protocols show different values of throughput by varying number of nodes. 


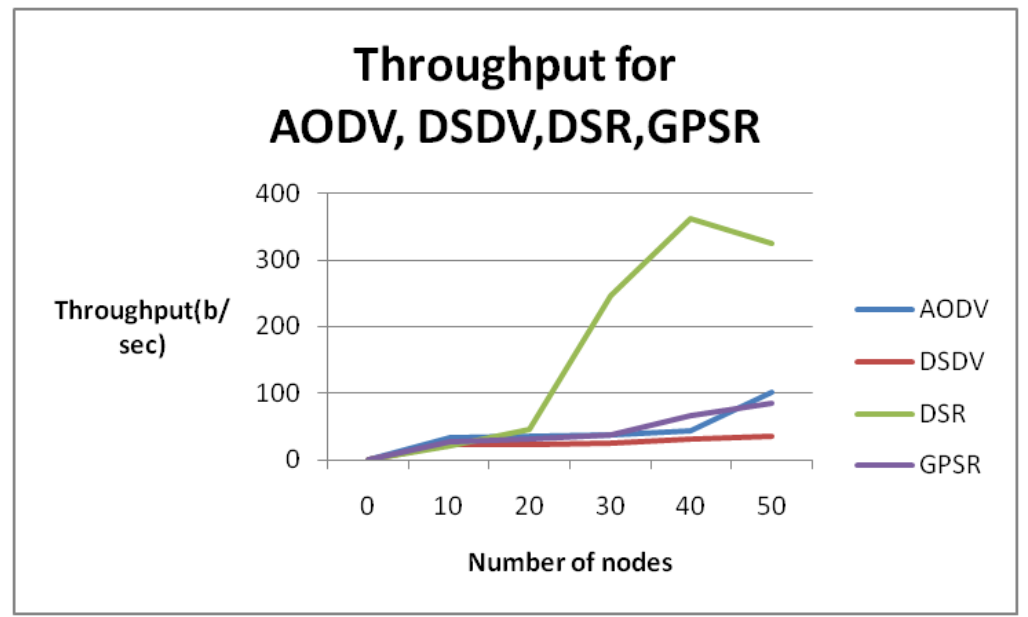

Figure 2: Impact of Throughput on Routing Protocols.

\subsection{Impact on End to End Delay}

Figure 3shows the impact of routing protocols on end to end Delay. More the value of End to End Delay less is the efficiency of protocol. Graph depicts that initially when the number of nodes was less then delay was almost similar in all protocols. As the number of nodes increased, delay reached a value of $376 \mathrm{msec}$ in AODV protocol, while all others show similar values. During simulations simulation time is fixed to $50 \mathrm{sec}$.

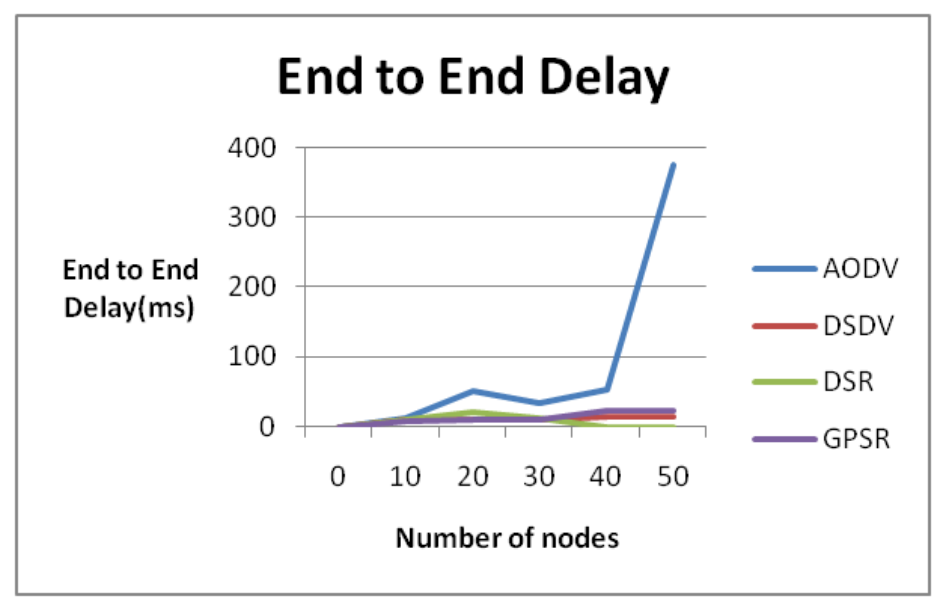

\subsection{Impact on Energy}

Figure 3: Impact of End to End Delay on Routing Protocols.

Energy is the value in unit of joules which shows how much energy is consumed by each node. Figure 3 shows the impact of routing protocols on energy and shows average energy consumed during each simulation carried out. Figure shows that initially when the number of nodes was 10 then energy value of DSDV is more as comparison to all other protocols. When the number of nodes increased, value of AODV reached more and at 50 nodes value of AODV and DSR is same while of GPSR is less. It is clearly depicted that among all four protocols GPSR consumes less energy than all other protocols and AODV use higher energy. Lower the energy consumed by protocol more is the performance of protocol. 


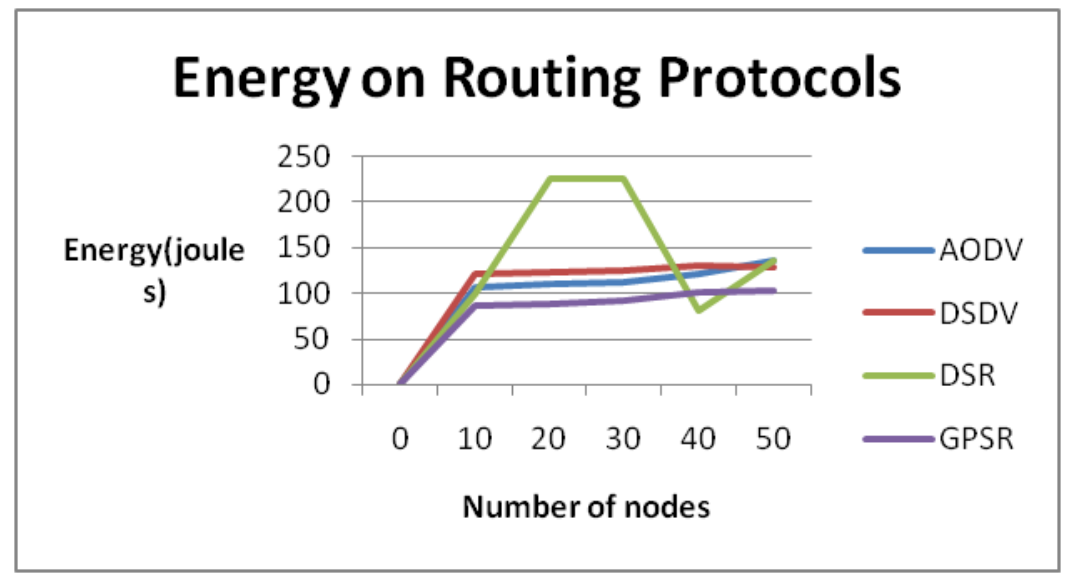

Figure 4: Impact of Energy on Routing Protocols.

\subsection{Impact on Packet Delivery Ratio}

The packets which are successfully transmitted can be calculated by Packet Delivery Ratio. Figure 5 shows the impact of routing protocol on packet Delivery Ratio. Initially when the number of nodes was less then GPSR shows a high value of PDR. As the number increased AODV seems to deliver more packets than all other protocols. At the end value of PDR in DSR reaches to 0 while DSDV and GPSR were found to deliver more packets. Simulation is carried out under environment where the number of nodes is varying and the simulation time is fixed to $50 \mathrm{sec}$.

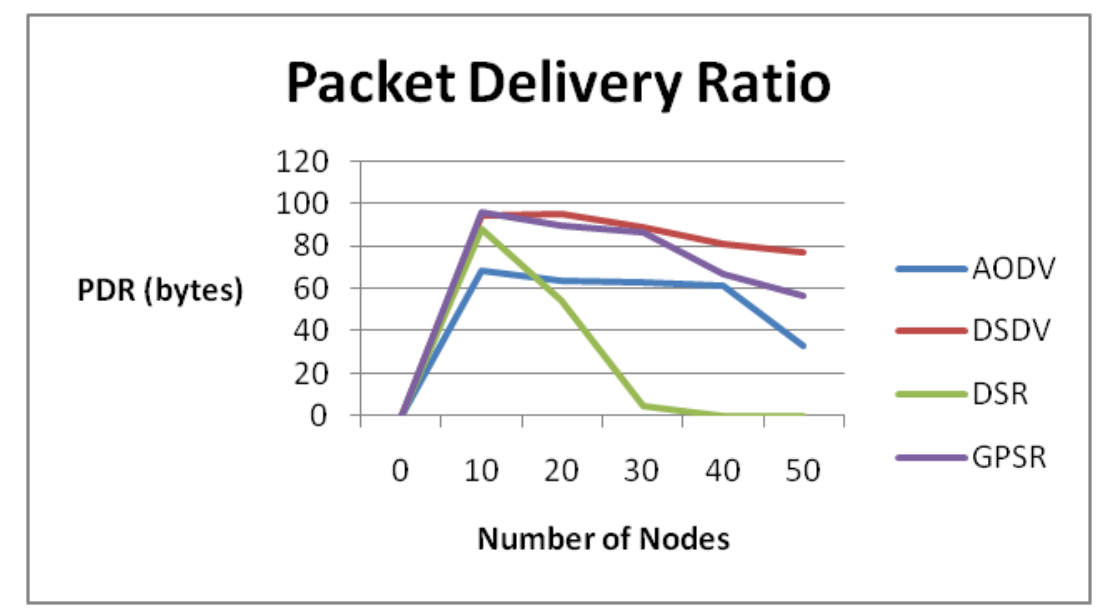

Figure 5: Impact of Routing Protocols on Packet Delivery Ratio.

\subsection{Impact on Routing Overhead}

Figure 6 shows the impact of routing protocols on overhead. DSR shows unusual behavior in case of routing overhead because initially when number of nodes was 10 or 20 overhead data was less than one and then at 30 it reaches to its peak. Among all other protocols DSDV shows less value of overhead. 


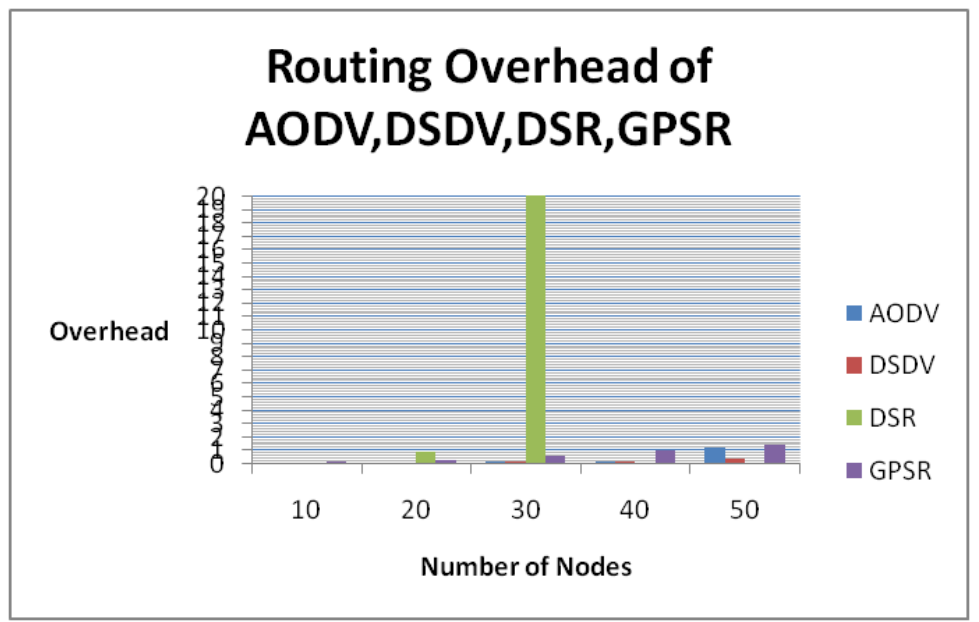

Figure 6: Routing Overhead.

\section{CONCLUSIONS}

In this paper, analysis based on simulation has been carried out in Vehicular Adhoc based scenario to analyze the network performance using different protocols under different network size. This work is based on Network simulator tool 2.35 which takes varying number of vehicles in every simulation. In this paper we have analyzed and compared position protocol, GPSR with topology based protocols AODV, DSR and DSDV on different parameters for Adhoc routing between V2V and V2I. Results shows that protocol behave differently on different parameters. While considering throughput AODV and GPSR performs better than other protocols and if energy is considered GPSR consumes less amount of energy than other protocols. End to End delay is more in DSR protocol while PDR is more in DSDV Protocol. In these ways different protocols shows better performance on various parameters. The simulated results may serve as guidelines for designing modern traffic control congestion system.

\section{REFERENCES}

1. http://www.who.int/news-room/fact-sheets/detail/road-traffic-injuries/

2. X. Ma, J. Zhang, X. Yin, K.S. Trivedi: Design and analysis of a robust broadcast scheme for VANET safety-related services.IEEE Trans. Veh. Technol. 61 (1), 46-61(2012).

3. M. Smita, N. Pathak: Secured communication in real time VANE. In: Proceedings of the International Conference on Emerging Trends in Engineering and Technology (ICETET) 2009, pp. 1151-1155, Nagpur, India, (2009).

4. A. Stampoulis, Z. Chai: A survey of security in vehicular networks. Project CPSC, vol. 534(2007).

5. Vishani, KM, and AA Bavarva. "Vanet Routing Protocol for Multihop Voice Transmission. "International Journal of Electronics and Communication Engineering (IJECE) 3. 3, May 2014, 81-86

6. N. S. Divya, VBobba: A theoretical Research on Routing Protocols for Vehicular AdHoc Networks. International Journal of Recent Technology and Engineering. 8(1S4), ISSN: 2277-3878(2019)

7. IEEE Draft Std., P802.11 p/D9.0, Sep. 2009 [Online], Available: http://ieeexplore.ieee.org/servlet/opac?pnumber-5325056.

8. S.K.Bhoi, P.M. Khilar: Vehicular communication: a survey. IET Netw.3(3), 204-217(2014)

9. E. M. Royer, C. E. Perkins: An implementation study of the AODV routing protocol. In: EEE Wireless Communications and Networking Conference. Conference Record (Cat. No.00TH8540), Chicago, IL, 2000, pp. 1003-1008 vol.3. (2000) 
10. Charan, P. I. Y. U. S. H., T. A. H. S. I. N. Usmani, and Syed Hasan Saeed. "A Comprehensive Study of Various on Demand Routing Protocols for MANETs." International Journal of Electronics and Communication Engineering 4 (2015): 1-12.

11. S.H Raut,H.P Ambulgaker: Proactive and reactive Routing Protocols in Multi Hop Mobile AdHoc Networks, IJRCSSE.3 (4).(2018)

12. S Malik, P. K Sahu: A Comparative Study on Routing Protocols in VANET.Elsevier, 5,pp.1-9,ISSN 2405-8440(2019)

13. H. Shahbazi, S. Jamshidinejad:Comparing three Protocols of DODV, DSR and DSDV for VANET Network. International Journal on Ad Hoc Networking Systems. 8(3).pp 1-10(2018).

14. Brad Karp, H.T. Kung: GPSR: Greedy Perimeter Stateless Routing Protocol. In: 6th Annual ACM/IEEE International Conference on Mobile Computing and Networking (MobiCom 2000).

15. Vineeth, Nandhini, and H. S. Guruprasad. "A survey on the techniques enhancing video streaming in VANETs." Int. J. Comput. Networking Wireless Mobile Commun 3 (2013): 37-46.

16. Silva, Andrey \& Reza, Niaz\& Oliveira: Aurenice: Improvement and Performance Evaluation of GPSR-Based Routing Techniques for Vehicular Ad Hoc Networks. IEEE Access. PP.(99).1-1(2019)

17. E.R. Cavalcanti, J.A.R.de-Souza, M.A Spohn, R.C. de-Morais Gomes, A.F.B.F. dacosta, VANET research over the past decade: overview, credibility and trends, SIGCOMM Comput. Commun. Rev. 48(2) (2018) 31-39.

18. Sharma, Vishal, Harsukhpreet Singh, and M. Kaur. "Implementation and Analysis of OFDM based IEEE $802.11 \mathrm{~g}$ VANET." IJCNWMC, Trans-Tellar (2013): 47-54.

19. S.Latif, S.Mahfooz, B.Jan, N.Ahmad, Y.Cao, M. Asif: A Comparative study of scenario-driven multi-hop broadcast protocols for VANET, J. Veh. Commun. 12, pp 88-109(2018) 
